\title{
HEREDITARY CIRCULARITY FOR ENERGY MINIMAL DIFFEOMORPHISMS
}

\author{
NGIN-TEE KOH
}

ABstract. We reveal some geometric properties of energy minimal diffeomorphisms defined on an annulus, whose existence was established in works by Iwaniec et al. (2011) and Kalaj (2014).

\section{INTRODUCTION}

Harmonic mappings between planar regions are generalizations of conformal mappings that satisfy the less restrictive Laplace equation in place of the CauchyRiemann equations. They possess some striking peculiarities, such as retaining an area-contraction property [18] while not necessarily decreasing either Euclidean or hyperbolic distances [7.

Energy minimal diffeomorphisms, which minimize the Dirichlet energy integral

$$
E(f)=\iint_{D}\left|f_{z}\right|^{2}+\left|f_{\bar{z}}\right|^{2}
$$

among all orientation-preserving homeomorphisms

$$
f: D \stackrel{\text { onto }}{\longrightarrow} \Omega
$$

with $E(f)<\infty$, form an important subclass of the family of harmonic mappings between $D$ and $\Omega$ [13, page 690] and have some interesting traits not necessarily shared by all harmonic mappings. Examples are properties related to hereditary convexity and hereditary circularity described in the next two paragraphs.

In the case where the domain $D$ from (2) is simply-connected, the energy minimal diffeomorphisms are conformal mappings [13, pages 668-669]. If $D$ is the unit disk $\mathbb{D}$, and if the target region $\Omega$ is convex, then the conformal image of every disk in $\mathbb{D}$ is also convex (see, e.g., [4, proof of Theorem 2.11] or [21]). This hereditary property, however, need not be present in harmonic mappings (see, e.g., [2, Example 5.5] or [5. pages 46-48]), which is due to the phenomenon that unidirectional convexity is not a hereditary property of conformal mappings (see, e.g., 2, Theorem 5.3], [6], [8]). Hence hereditary convexity is not a generic attribute of harmonic mappings.

In the special case where $D=\Omega=\mathbb{D}$, the energy minimal diffeomorphisms are Möbius transformations, and thus the conformal image of every disk in $\mathbb{D}$ is again a disk. Once again, however, harmonic mappings do not necessarily possess this hereditary property. It was shown in [1, Proposition 2] a harmonic mapping on a simply-connected domain that maps circles to ellipses has constant dilatation. The existence of harmonic self-mappings of $\mathbb{D}$ with non-constant dilatation was already

Received by the editors November 1, 2016, and, in revised form, August 20, 2017.

2010 Mathematics Subject Classification. Primary 30C45, 31A05, 34B24.

Key words and phrases. Annulus, energy minimal diffeomorphisms, Sturm-Liouville theory. 
established in [9, Theorem 4.2]. Hence harmonic self-mappings of $\mathbb{D}$ need not map a disk to another disk.

In the doubly-connected case where $D$ is the annulus

$$
\mathbb{A}_{\rho}=\{z \in \mathbb{C}: 0<\rho<|z|<1\}
$$

and where $\Omega$ is a bounded doubly-connected region, an energy minimal diffeomorphism $h: \mathbb{A}_{\rho} \longrightarrow \Omega$ exists provided the conformal modulus of $\mathbb{A}_{\rho}$ is not greater than that of $\Omega$ (see, e.g., [13, Theorem 1.1] or [15, Theorem 1.1]). If the boundary components of $\Omega$ are convex Jordan curves, then a recent result [16, Theorem 3.5] shows that $h\left(\mathbb{T}_{r}\right)$ is a strictly convex curve for each $r \in(\rho, 1)$, where

$$
\mathbb{T}_{r}=\{z \in \mathbb{C}:|z|=r\} .
$$

The same conclusion holds for a wider class of harmonic mappings $f$ that satisfy

$$
\Delta \operatorname{Im} \log \left(1-\frac{\bar{z} f_{\bar{z}}}{z f_{z}}\right)=0
$$

on $\mathbb{A}_{\rho}$, where $\Delta$ represents the Laplace operator [16, Theorem 2.2]. There is a similar result when the boundary components of $\Omega$ are starlike Jordan curves [17. In this article, we will demonstrate among other things that if the boundary components of $\Omega$ are circles, then $h\left(\mathbb{T}_{r}\right)$ is a circle for each $r \in(\rho, 1)$.

\section{MAIN RESUlT}

Let $\mathbb{T}$ denote the unit circle $\partial \mathbb{D}$. The following result about energy minimal diffeomorphisms will be established.

Theorem 2.1. Let $h: \mathbb{A}_{\rho} \stackrel{\text { onto }}{\longrightarrow} \Omega$ be an energy minimal diffeomorphism, where $\Omega$ is a bounded doubly-connected region whose boundary components are circles. Then $h\left(\mathbb{T}_{r}\right)$ is a circle for $\rho<r<1$.

Theorem 2.1 raises the following question.

Open Problem 2.2. Let $h: \mathbb{A}_{\rho} \stackrel{\text { onto }}{\longrightarrow} \Omega$ be a harmonic mapping, where $\Omega$ is a bounded doubly-connected region whose boundary components are circles. Is $h\left(\mathbb{T}_{r}\right)$ a circle for each $r \in(\rho, 1)$ ?

Remark 2.3. In the special case where $\Omega$ in Open Problem 2.2 is an annulus $\mathbb{A}_{\sigma}$ for some $\sigma>0$ (so that the boundary circles are concentric), it is known that the harmonic images $h\left(\mathbb{T}_{r}\right)$ of concentric circles need not be concentric circles (see, e.g., [10, page 170] and [16, page 234]). However, if $h$ is also an energy minimal diffeomorphism, then concentric circles are necessarily mapped to concentric circles [14, Theorem 1.5].

\section{HARMONICITY}

The proof of Theorem 2.1 derives from certain arguments used in establishing the following lemma, which may be of independent interest. Its proof is deferred to Section 5.1 .

Lemma 3.1. Let $h: \mathbb{A}_{\rho} \stackrel{\text { onto }}{\longrightarrow} \Omega$ be an energy minimal diffeomorphism, where $\Omega$ is a bounded doubly-connected region, and let $z=r e^{i t} \in \mathbb{A}_{\rho}$. If $h$ is not conformal, and if $s$ represents an arc length parameter on $h\left(\mathbb{T}_{r}\right)$, then there is a non-zero constant $k \in \mathbb{C}$ that is either positive or purely imaginary such that $\operatorname{arccosh} k \frac{\partial s}{\partial t}$ is a single-valued harmonic function on $\mathbb{A}_{\rho}$. 
Remark 3.2. The harmonic function $V=\operatorname{arccosh} k \frac{\partial s}{\partial t}$ from Lemma 3.1 is related to the curvature of $h\left(\mathbb{T}_{r}\right)$ by condition (16) below, and plays an important role in the proof of Theorem 2.1. The harmonicity of $V$, in particular, is used to arrive at the differential equations (18) and (20).

\section{BOUNDARY BEHAVIOR}

Let $\theta$ and $\phi$ denote polar angles. In this section, we study the boundary behavior of a harmonic mapping between an annulus $\mathbb{A}_{\rho}$ and a doubly-connected region $\Omega$ bounded by two Jordan curves. We first recall a result from [16, Proposition 4.1] that had its origins pertaining to simply-connected regions in [9, Theorem 4.3].

Proposition 4.1. Let $h$ be a harmonic mapping of $\mathbb{A}_{\rho}$ into a doubly-connected region $\Omega$ bounded by two Jordan curves $C_{1}$ and $C_{\rho}$ in the finite plane such that the radial limits $\lim _{r \rightarrow 1} h\left(r e^{i \theta}\right)$ and $\lim _{r \rightarrow \rho} h\left(r e^{i \phi}\right)$ lie on $C_{1}$ and $C_{\rho}$, respectively, for almost all $\theta$ and $\phi$. Then there is a countable set $W \subset \partial \mathbb{A}_{\rho}=\mathbb{T} \cup \mathbb{T}_{\rho}$ such that the unrestricted limits

$$
H\left(e^{i \theta}\right)=\lim _{z \rightarrow e^{i \theta}} h(z), \quad H\left(\rho e^{i \phi}\right)=\lim _{z \rightarrow \rho e^{i \phi}} h(z)
$$

exist on $\partial \mathbb{A}_{\rho} \backslash W$, and are contained in $C_{1}, C_{\rho}$, respectively. Moreover,

(a) $H$ is both continuous and orientation-preserving on $\mathbb{T} \backslash W$ and $\mathbb{T}_{\rho} \backslash W$;

(b) for each $e^{i \theta} \in W$, the one-sided limits

$$
H\left(e^{i \theta-}\right)=\lim _{\substack{\sigma \rightarrow \theta^{-} \\ e^{i \sigma} \notin W}} H\left(e^{i \sigma}\right), \quad H\left(e^{i \theta+}\right)=\lim _{\substack{\sigma \rightarrow \theta^{+} \\ e^{i \sigma} \notin W}} H\left(e^{i \sigma}\right),
$$

exist, belong to $C_{1}$, and are distinct;

(c) for each $\rho e^{i \phi} \in W$, the one-sided limits

$$
H\left(\rho e^{i \phi-}\right)=\lim _{\substack{\sigma \rightarrow \phi^{-} \\ \rho e^{i \sigma} \notin W}} H\left(\rho e^{i \sigma}\right), \quad H\left(\rho e^{i \phi+}\right)=\lim _{\substack{\sigma \rightarrow \phi^{+} \\ \rho e^{i \sigma} \notin W}} H\left(\rho e^{i \sigma}\right)
$$

exist, belong to $C_{\rho}$, and are distinct;

(d) the cluster set of $h$ at the points $e^{i \theta} \in W$ and $\rho e^{i \phi} \in W$ are the line segments joining $H\left(e^{i \theta-}\right)$ to $H\left(e^{i \theta+}\right)$ and $H\left(\rho e^{i \phi-}\right)$ to $H\left(\rho e^{i \phi+}\right)$, respectively.

Suppose $f$ is a harmonic mapping of $\mathbb{A}_{\rho}$ onto a doubly-connected region $\Omega$ bounded by two Jordan curves in the finite plane. By Fatou's Theorem (see, e.g., [11, 2nd corollary on page 38]), the radial limits $\lim _{r \rightarrow 1} f\left(r e^{i \theta}\right)$ and $\lim _{r \rightarrow \rho} f\left(r e^{i \phi}\right)$ of the bounded function $f$ exist almost everywhere, and are contained in distinct boundary components of $\Omega$ since $f$ is a homeomorphism (see, e.g., [19, page 11]). It follows from Proposition 4.1 that $f$ may be extended continuously to $\partial \mathbb{A}_{\rho}$ outside a countable set $W$, and that each boundary point of $\Omega$ corresponds to a non-empty "pre-image" (with infinitely many points on a boundary line segment of $\Omega$ associated with a "pre-image" point in $W$ from (c)). Hence $W$ will be empty if $\Omega$ contains no line segment, in which case $f$ has a continuous extension to $\partial \mathbb{A}_{\rho}$, and we have

Corollary 4.2. Let $h$ be a harmonic mapping of $\mathbb{A}_{\rho}$ onto a doubly-connected region $\Omega$ bounded by two Jordan curves that do not contain line segments. Then $h$ has a continuous extension to $\partial \mathbb{A}_{\rho}$. 


\section{Derivatives in LOG-POlar COORDinates}

Let $h: \mathbb{A}_{\rho} \stackrel{\text { onto }}{\longrightarrow} \Omega$ be an energy minimal diffeomorphism, where $\Omega$ is a bounded doubly-connected region. It was shown in [13, Lemma 6.1] that

$$
h_{z} \overline{h_{\bar{z}}}=\frac{c}{z^{2}},
$$

where $c$ is a real constant. Energy minimal diffeomorphims are the diffeomorphic solutions to (44). Let

$$
u=\ln r, \quad v=t,
$$

so that we may write

$$
z=r e^{i t}=e^{u+i v} .
$$

The complex derivatives $h_{z}$ and $h_{\bar{z}}$ in logarithmic polar coordinates are

$$
h_{z}=\frac{e^{-i t}}{2 r}\left(h_{u}-i h_{v}\right), \quad h_{\bar{z}}=\frac{e^{i t}}{2 r}\left(h_{u}+i h_{v}\right) .
$$

Hence

$$
h_{z} \overline{h_{\bar{z}}}=\frac{e^{-i 2 t}}{4 r^{2}}\left(\left|h_{u}\right|^{2}-\left|h_{v}\right|^{2}-i 2 \operatorname{Re} h_{u} \overline{h_{v}}\right) .
$$

Taking the real and imaginary parts of the expression in parentheses in (6) and comparing them with (4), and then writing

$$
h=x+i y,
$$

we obtain

$$
x_{u}^{2}+y_{u}^{2}-x_{v}^{2}-y_{v}^{2}=4 c
$$

and

$$
x_{u} x_{v}+y_{u} y_{v}=0
$$

Let

$$
w=-i(u+i v)=v-i u \text {. }
$$

For each $w$, we may take a disk $D$ in $\mathbb{A}_{\rho}$ containing $w$ such that $x=\operatorname{Re} f$ and $y=\operatorname{Re} g$ for some holomorphic functions $f$ and $g$ defined on $D$. In conjunction with (9), we may rewrite (8) locally as

$$
f^{\prime}(w)^{2}+g^{\prime}(w)^{2}=-4 c .
$$

Hence

$$
f^{\prime}(w)=\sqrt{-4 c} \cos (U+i V), \quad g^{\prime}(w)=\sqrt{-4 c} \sin (U+i V),
$$

where $U+i V$ is a holomorphic function of $w$. In view of (10), this yields

$$
\begin{array}{ll}
x_{v}=\sqrt{-4 c} \cos U \cosh V, & x_{u}=-\sqrt{-4 c} \sin U \sinh V, \\
y_{v}=\sqrt{-4 c} \sin U \cosh V, & y_{u}=\sqrt{-4 c} \cos U \sinh V,
\end{array}
$$

from which we obtain

$$
\sqrt{-4 c} \cosh V=\sqrt{x_{v}^{2}+y_{v}^{2}}, \quad \tanh V=\frac{y_{u}}{x_{v}}=-\frac{x_{u}}{y_{v}},
$$

and

$$
\cos U=\frac{x_{v}}{\sqrt{x_{v}^{2}+y_{v}^{2}}}, \quad \sin U=\frac{y_{v}}{\sqrt{x_{v}^{2}+y_{v}^{2}}}
$$


It follows from (5), (17), and (12) that the harmonic function

$$
U \equiv \arg \frac{\partial h}{\partial v} \equiv \arg \frac{\partial h}{\partial t} \bmod 2 \pi .
$$

We mention in passing that while $\arg \frac{\partial h}{\partial t}$ satisfies Laplace's equation for a more general class $\mathcal{H}$ of harmonic mappings for which (3) holds (see, e.g., [16, page 240]), the above argument does not apply to functions in $\mathcal{H}$. For instance, it was shown in [16, page 234] certain harmonic mappings taking the form

$$
h(z)=\frac{z+a}{1+a z}-b \log |z|,
$$

where $a \in(0,1)$ and $b=\frac{a\left(1-\rho^{2}\right)}{\left(1-\rho^{2} a^{2}\right) \log \rho}<0$, are in $\mathcal{H}$. However, the Hopf differentials of such $h$ are given by

$$
h_{z} \overline{h_{\bar{z}}}=\frac{b^{2}}{4 z^{2}}-\frac{\left(1-a^{2}\right) b}{2 z(1+a z)^{2}},
$$

which is different from (4).

5.1. Proof of Lemma 3.1, It is clear from (4) that conformality of $h$ is characterized by the condition $c=0$. Hence we restrict our attention to the case where $c \neq 0$. Observe that the principal value of

$$
\operatorname{arccosh} z=\log [z+\sqrt{z+1} \sqrt{z-1}]
$$

is defined for $z \in \mathbb{C} \backslash(-\infty, 1]$. Recall that if $s$ is an arc length parameter on $h\left(\mathbb{T}_{r}\right)$, then

$$
\frac{\partial s}{\partial v}=\sqrt{x_{v}^{2}+y_{v}^{2}}
$$

If $c<0$, then since

$$
x_{v}^{2}+y_{v}^{2}>-4 c>0
$$

from (8), we have

$$
\sqrt{\frac{x_{v}^{2}+y_{v}^{2}}{-4 c}}>1
$$

If $c>0$, then

$$
\arg \sqrt{\frac{x_{v}^{2}+y_{v}^{2}}{-4 c}} \in\left\{(2 n-1) \frac{\pi}{2}: n \in \mathbb{Z}\right\}
$$

Hence

$$
\sqrt{\frac{x_{v}^{2}+y_{v}^{2}}{-4 c}} \in \mathbb{C} \backslash(-\infty, 1]
$$

if $c \neq 0$, and it follows from (11) and (14) that the single-valued function

$$
V=\operatorname{arccosh} \frac{1}{\sqrt{-4 c}} \frac{\partial s}{\partial v}=\operatorname{arccosh} \sqrt{\frac{x_{v}^{2}+y_{v}^{2}}{-4 c}}
$$

is harmonic, being locally the imaginary part of the holomorphic function $U+i V$. In view of (5), taking $k=\frac{1}{\sqrt{-4 c}}$ establishes Lemma 3.1. 


\section{Curvature considerations}

We recall the variables $U, V, s, u, v$, and $w$ from the previous section. The curvature of $h\left(\mathbb{T}_{r}\right)$ is given by

$$
K(u, v)=\frac{d U}{d s}=\frac{\frac{\partial U}{\partial v}}{\frac{\partial s}{\partial v}}=\frac{\operatorname{sech} V}{-\sqrt{-4 c}} \frac{\partial V}{\partial u}
$$

from the Cauchy-Riemann equations and (15). Partial differentiation with respect to $v$ yields

$$
\frac{\partial K}{\partial v}=\frac{\operatorname{sech} V}{-\sqrt{-4 c}}\left(\frac{\partial^{2} d}{\partial v \partial u}-\tanh V \frac{\partial V}{\partial v} \frac{\partial V}{\partial u}\right)
$$

Let

$$
A(u, v)=-\sqrt{-4 c} \cosh V \frac{\partial K}{\partial v}=\frac{\partial^{2} V}{\partial v \partial u}-\tanh V \frac{\partial V}{\partial v} \frac{\partial V}{\partial u} .
$$

The harmonicity of $V$ implies that the expressions $\frac{\partial V}{\partial u}, \frac{\partial V}{\partial v}$, and $\frac{\partial^{2} V}{\partial v \partial u}$ all satisfy Laplace's equation. A lengthy calculation shows that

$$
\frac{\partial^{2} A}{\partial u^{2}}+\frac{\partial^{2} A}{\partial v^{2}}+2\left[\left(\frac{\partial V}{\partial u}\right)^{2}+\left(\frac{\partial V}{\partial v}\right)^{2}\right]\left(\operatorname{sech}^{2} V\right) A=0 .
$$

6.1. A change of variables. Suppose $h: \mathbb{A}_{\rho} \stackrel{\text { onto }}{\longrightarrow} \Omega$ is an energy minimal diffeomorphism, where $\Omega$ is a bounded doubly-connected region whose boundary components are circles. Since $\Omega$ is bounded by convex Jordan curves, it follows from [16. Theorem 3.5] that $h\left(\mathbb{T}_{r}\right)$ is strictly convex for $r \in(\rho, 1)$. This means from (5) and (13) that

$$
\frac{\partial U}{\partial v}=\frac{\partial}{\partial t} \arg \frac{\partial h}{\partial t}>0
$$

on $\mathbb{A}_{\rho}$.

Let $Z=e^{i(U+i V)}$, which is a holomorphic function of

$$
z=e^{u+i v}=e^{i w} \in \mathbb{A}_{\rho} .
$$

It follows from (19) that $\frac{d Z}{d z} \neq 0$. Hence the mapping $f(z)=Z$ of $\mathbb{A}_{\rho}$ is locally univalent and non-zero.

Consider the logarithmic polar coordinates $(-V, U)$ in the $Z$-plane. The inequality (19) means that the pre-image under $f$ of a ray defined by any fixed $U$ is a curve that intersects each concentric circle $\mathbb{T}_{r}$ exactly once, and non-tangentially. The disjoint union of all such pre-image curves of $U=c$, where $c \in[0,2 \pi)$, contains $\mathbb{A}_{\rho}$.

Let $l$ be an arc length parameter on a pre-image curve $C$ of $U=c$, and let $n$ denote a parameter that varies monotonically in a direction normal to $C$. Then (19) implies that

$$
\left|\frac{\partial V}{\partial l}\right|=\left|\frac{\partial U}{\partial n}\right| \neq 0
$$

so $V$ is monotone on $C$.

Each curve $C \cap \mathbb{A}_{\rho}$ is mapped by $f$ to a ray segment in the $Z$-plane whose endpoints have bounded non-zero polar distance from the origin. Hence there is a bounded non-degenerate annulus $S$ centred at the origin containing the image $R$ 
of $\mathbb{A}_{\rho}$ in the $Z$-plane. In logarithmic polar-coordinates, there exist real numbers $V_{1}<V_{2}$ such that

$$
S=\left\{Z: e^{-V_{2}}<|Z|<e^{-V_{1}}\right\}
$$

Hence $A$ is a function of the variables $U$ and $V$ in the $Z$-plane, as well as being a function of the variables $u$ and $v$ in the $z$-plane. With the aid of the Lamé coefficients $\left|\frac{\partial V}{\partial u}\right|$ and $\left|\frac{\partial V}{\partial v}\right|$, we may write

$$
\frac{\partial^{2} A}{\partial u^{2}}+\frac{\partial^{2} A}{\partial v^{2}}=\left[\left(\frac{\partial V}{\partial u}\right)^{2}+\left(\frac{\partial V}{\partial v}\right)^{2}\right]\left(\frac{\partial^{2} A}{\partial U^{2}}+\frac{\partial^{2} A}{\partial V^{2}}\right),
$$

and obtain, from (18),

$$
\frac{\partial^{2} A}{\partial U^{2}}+\frac{\partial^{2} A}{\partial V^{2}}+2\left(\operatorname{sech}^{2} V\right) A=0 .
$$

6.2. Proof of Theorem 2.1. Let $h: \mathbb{A}_{\rho} \stackrel{\text { onto }}{\longrightarrow} \Omega$ be an energy minimal diffeomorphism, where $\Omega$ is a bounded doubly-connected region whose boundary components are circles. By Corollary $4.2, h$ has a continuous extension to $\partial \mathbb{A}_{\rho}$. Since the curvature $K(u, v)$ (from (16)) is constant for the boundary circles of $h\left(\mathbb{A}_{\rho}\right)=\Omega$, it follows that $\frac{\partial K}{\partial v}=0$ on $\partial \mathbb{A}_{\rho}$ and thus, from (17), we have

$$
A=0 \text { on } \partial \mathbb{A}_{\rho} \text {. }
$$

It suffices to show that $A \equiv 0$ on $\mathbb{A}_{\rho}$, for then $\frac{\partial K}{\partial v} \equiv 0$ on $\mathbb{A}_{\rho}$ so that $K$ is constant on $\mathbb{T}_{r}$ for each $r$, which would mean that $h\left(\mathbb{T}_{r}\right)$ is a circle for each $r$, as stated in Theorem 2.1. Suppose to the contrary that

$$
A \not \equiv 0 \text { on } \mathbb{A}_{\rho} \text {. }
$$

Since $K$ is a single-valued function on $\mathbb{A}_{\rho}$,

$$
-\sqrt{-4 c} \int_{0}^{2 \pi} \frac{\partial K}{\partial v} d v=\int_{0}^{2 \pi} \frac{A}{\cosh V} d v=0 .
$$

Hence $A$ changes signs on $\mathbb{A}_{\rho}$ and, in the $Z$-plane, we have

$$
A=0 \text { on } \partial R, \quad A \text { changes signs on } R \text {. }
$$

Related to (20) are two eigenvalue problems

$$
\frac{\partial^{2} B}{\partial U^{2}}+\frac{\partial^{2} B}{\partial V^{2}}+\left[2\left(\operatorname{sech}^{2} V\right)+\lambda\right] B=0 \text { on } R, \quad B=0 \text { on } \partial R,
$$

and

$$
\frac{\partial^{2} B}{\partial U^{2}}+\frac{\partial^{2} B}{\partial V^{2}}+\left[2\left(\operatorname{sech}^{2} V\right)+\mu\right] B=0 \text { on } S, \quad B=0 \text { on } \partial S .
$$

In view of (20) and (22), we make the following observations from two-dimensional Sturm-Liouville theory (see, e.g., [3, page 452 and page 455 footnote 1]) for the eigenvalue problem in (23):

- $A$ is an eigenfunction that is not the first eigenfunction;

- $A$ is associated with the eigenvalue $\lambda=0$.

Denoting the eigenvalues for the eigenvalue problem in (23) by

$$
\lambda_{1}<\lambda_{2}<\ldots<\lambda_{n}<\ldots,
$$

this means that

$$
\lambda_{2} \leq 0 .
$$


Denoting the eigenvalues for the eigenvalue problem in (24) by

$$
\mu_{1}<\mu_{2}<\ldots<\mu_{n}<\ldots,
$$

we have

$$
\mu_{n}<\lambda_{n} \text { for all } n \in \mathbb{N}
$$

since enlarging the domain reduces the corresponding eigenvalues (see, e.g., [3, page 409, Theorem 3]). Separating variables in (24) yields that a complete system of eigenfunctions associated with $\mu_{n}$ is given by

$$
\left(c_{k} \cos k U+d_{k} \sin k U\right) Q_{k, n}(V), \quad k \in\{0\} \cup \mathbb{N},
$$

where $Q_{k, n}(V)$ is a solution to the eigenvalue problem

$$
\begin{aligned}
Q_{k, n}^{\prime \prime}+\left[2\left(\operatorname{sech}^{2} V\right)+\nu_{k, n}\right] Q_{k, n} & =0 & & \text { if } V \in\left(V_{1}, V_{2}\right), \\
Q_{k, n} & =0 & & \text { if } V \in\left\{V_{1}, V_{2}\right\},
\end{aligned}
$$

and $\nu_{k, n}=\mu_{n}-k^{2}$. Reordering and denoting these eigenvalues by

$$
\nu_{1}<\nu_{2}<\ldots<\nu_{m}<\ldots,
$$

we see that the eigenvalues $\mu_{n}$ may be listed in terms of $\nu_{m}$ as follows:

$$
\nu_{1}<\nu_{1}+1<\ldots<\nu_{2}<\ldots
$$

If $\nu_{m}=-1$, then a solution to (27) is given by $\operatorname{sech} V$, which is non-zero for all $V \in \mathbb{R}$. We deduce from the Sturm separation theorem (see, e.g., [12, page 224]) that any solution to (27) when $\nu_{k, n}=-1$ will have at most one zero on $\mathbb{R}$ and violate (28). By Sturm-Liouville theory (see, e.g., [3, pages 454-455] or [12, pages $231-235])$,

$$
\nu_{1}>-1 \text {. }
$$

In view of (26), (29), and (30), this yields

$$
\lambda_{2}>\mu_{2}=\nu_{1}+1>0,
$$

a contradiction to (25) that can be traced back to assumption (21). This proves Theorem 2.1.

\section{REFERENCES}

[1] Martin Chuaqui, Peter Duren, and Brad Osgood, Ellipses, near ellipses, and harmonic Möbius transformations, Proc. Amer. Math. Soc. 133 (2005), no. 9, 2705-2710. MR2146217

[2] J. Clunie and T. Sheil-Small, Harmonic univalent functions, Ann. Acad. Sci. Fenn. Ser. A I Math. 9 (1984), 3-25. MR752388

[3] R. Courant and D. Hilbert, Methods of mathematical physics. Vol. I, Interscience Publishers, Inc., New York, N.Y., 1953. MR0065391

[4] Peter L. Duren, Univalent functions, Grundlehren der Mathematischen Wissenschaften [Fundamental Principles of Mathematical Sciences], vol. 259, Springer-Verlag, New York, 1983. MR708494

[5] Peter Duren, Harmonic mappings in the plane, Cambridge Tracts in Mathematics, vol. 156, Cambridge University Press, Cambridge, 2004. MR2048384

[6] A. W. Goodman and E. B. Saff, On univalent functions convex in one direction, Proc. Amer. Math. Soc. 73 (1979), no. 2, 183-187. MR516461

[7] Erhard Heinz, On one-to-one harmonic mappings, Pacific J. Math. 9 (1959), 101-105. MR.0104933

[8] W. Hengartner and G. Schober, A remark on level curves for domains convex in one direction, Applicable Anal. 3 (1973), 101-106. Collection of articles dedicated to Eberhard Hopf on the occasion of his 70 th birthday. MR0393450 
[9] W. Hengartner and G. Schober, Harmonic mappings with given dilatation, J. London Math. Soc. (2) 33 (1986), no. 3, 473-483. MR 850963

[10] Walter Hengartner and Glenn Schober, Univalent harmonic exterior and ring mappings, J. Math. Anal. Appl. 156 (1991), no. 1, 154-171. MR 1102603

[11] Kenneth Hoffman, Banach spaces of analytic functions, Prentice-Hall Series in Modern Analysis, Prentice-Hall, Inc., Englewood Cliffs, N. J., 1962. MR0133008

[12] E. L. Ince, Ordinary Differential Equations, Dover Publications, New York, 1944. MR0010757

[13] Tadeusz Iwaniec, Ngin-Tee Koh, Leonid V. Kovalev, and Jani Onninen, Existence of energyminimal diffeomorphisms between doubly connected domains, Invent. Math. 186 (2011), no. 3, 667-707. MR2854087

[14] Tadeusz Iwaniec, Leonid V. Kovalev, and Jani Onninen, The Nitsche conjecture, J. Amer. Math. Soc. 24 (2011), no. 2, 345-373. MR2748396

[15] David Kalaj, Energy-minimal diffeomorphisms between doubly connected Riemann surfaces, Calc. Var. Partial Differential Equations 51 (2014), no. 1-2, 465-494. MR3247397

[16] Ngin-Tee Koh, Hereditary convexity for harmonic homeomorphisms, Indiana Univ. Math. J. 64 (2015), no. 1, 231-243. MR 3320525

[17] Ngin-Tee Koh, Harmonic mappings with hereditary starlikeness, J. Math. Anal. Appl. 457 (2018), no. 1, 273-286. MR.3702706

[18] N.-T. Koh and L. V. Kovalev, Area contraction for harmonic automorphisms of the disk, Bull. Lond. Math. Soc. 43 (2011), no. 1, 91-96. MR2765553

[19] O. Lehto and K. I. Virtanen, Quasiconformal mappings in the plane, 2nd ed., Springer-Verlag, New York-Heidelberg, 1973. Translated from the German by K. W. Lucas; Die Grundlehren der mathematischen Wissenschaften, Band 126. MR0344463

[20] Zeev Nehari, Conformal mapping, McGraw-Hill Book Co., Inc., New York, Toronto, London, 1952. MR0045823

[21] E. Study, Vorlesungen über ausgewählte Gegenstände der Geometrie, Heft II, Teubner, Leipzig, 1913.

School of Mathematics and Statistics, University of Canterbury, Private Bag 4800, Christchurch 8140, New Zealand

Email address: ngin-tee.koh@canterbury.ac.nz 